\title{
Submodular Flow Problem with a Nonseparable Cost Function*
}

\author{
Kazuo Murota ${ }^{\dagger}$
}

\begin{abstract}
The submodular flow problem is extended by considering a nonseparable cost function, which is assumed to enjoy a variant of the exchange property of the base polyhedron of a submodular system. Two optimality criteria are established, one in terms of potentials associated with vertices and the other in terms of negative cycles in an auxiliary graph. These are natural extensions of the well-known result for the conventional min-cost flow problem as well as the recent result of Fujishige for the submodular flow problem with a separable convex cost function.
\end{abstract}

(Key words.) submodular flow problem, valuated matroid, negative cycle, combinatorial optimization.

(AMS subject classifications.) 90C35; 90C27, 90C10.

March 1995; Revised January 1996 (Version: April 17, 1996)

Address for correspondence:

Kazuo Murota

Research Institute for Mathematical Sciences

Kyoto University, Kyoto 606-01, Japan

phone: +81-75-753-7221, facsimile: +81-75-753-7272

e-mail: murota@kurims.kyoto-u.ac.jp

*RIMS preprint 1061. Revised version of Report No. 95843-OR, Forschungsinstitut für Diskrete Mathematik, Universität Bonn, March 1995.

${ }^{\dagger}$ Research Institute for Mathematical Sciences, Kyoto University, Kyoto 606-01, Japan, e-mail: murota@kurims.kyoto-u.ac.jp. This work was done while the author was at Forschungsinstitut für Diskrete Mathematik, Universität Bonn, 1995. 


\section{Introduction}

The submodular flow problem introduced by Edmonds-Giles [9] is one of the most powerful and beautiful frameworks in combinatorial optimization. Along with other equivalent frameworks such as the independent flow problem (Fujishige [15]) and the polymatroidal flow problem (Hassin [20], Lawler-Martel [21]), it includes as special cases many other important problems such as the minimum cost flow problem, the (poly)matroid intersection problem (Edmonds [8]), the graph orientation problem (Frank [12]), and the directed cut covering problem (Lucchesi-Younger [22]). It may safely be said that the structure of the submodular flow problem is understood completely with a variety of efficient algorithms (Cunningham-Frank [2], Frank [12], Fujishige-Röck-Zimmermann [18], Gabow [19], Schönsleben [33], Zimmermann [37]). See Bixby-Cunningham [1], Faigle [10], Frank [13], FrankTardos [14], Fujishige [17], Nemhauser-Rinnooy Kan-Todd [32], Schrijver [34] for background materials.

The (integral) submodular flow problem is described as follows. Let $G=(V, A)$ be a graph with a vertex set $V$ and an $\operatorname{arc}$ set $A$. We are given an upper capacity function $\bar{c}: A \rightarrow \mathbf{Z} \cup\{+\infty\}$, a lower capacity function $\underline{c}: A \rightarrow \mathbf{Z} \cup\{-\infty\}$, and a cost function $\gamma: A \rightarrow R$, where $R$ is a totally ordered additive group ${ }^{1}$ (typically $R=\mathbf{R}$ (reals), $\mathbf{Q}$ (rationals), or $\mathbf{Z}$ (integers)). Furthermore, we are given (the integral points of) the base polyhedron $B \subseteq \mathbf{Z}^{V}$ of an integral submodular system. For a flow $\varphi: A \rightarrow \mathbf{Z}$ we define its boundary $\partial \varphi: V \rightarrow \mathbf{Z}$ by

$$
\partial \varphi(v)=\sum\left\{\varphi(a) \mid a \in \delta^{+} v\right\}-\sum\left\{\varphi(a) \mid a \in \delta^{-} v\right\}
$$

where $\delta^{+} v$ and $\delta^{-} v$ denote the sets of the out-going and in-coming arcs incident to $v$, respectively. Note that $\partial \varphi(V) \equiv \sum\{\partial \varphi(v) \mid v \in V\}=0$.

\section{[Submodular flow problem (P0)]}

Minimize

$$
\Gamma_{0}(\varphi)=\sum_{a \in A} \gamma(a) \varphi(a)
$$

subject to

$$
\begin{aligned}
& \underline{c}(a) \leq \varphi(a) \leq \bar{c}(a) \quad(a \in A), \\
& \partial \varphi \in B .
\end{aligned}
$$

Remark 1.1 In the original definition of the submodular flow problem, $B$ is assumed to be given in terms of a crossing-submodular function. It has been pointed

\footnotetext{
${ }^{1}$ Though we follow the standard setting [17], [36] to employ a rather abstract terminology, the reader may be advised to assume that $R$ represents $\mathbf{R}$ or $\mathbf{Z}$.
} 
out by Fujishige [16] that such polyhedron can be obtained as the base polyhedron of a submodular system (cf. Fujishige [17] for the definition of a submodular system). Since we do not discuss issues like algorithms and total dual integrality that depend on the description of $B$, we do not lose generality by assuming that $B$ is the base polyhedron of a submodular system. Moreover, we need not refer to the submodular function at all in this paper. What we rely on is the exchange property that characterizes the base polyhedron of a submodular system.

On the other hand, Dress-Wenzel [6], [7] introduced the concept of a valuated matroid, as a quantitative generalization of the concept of matroid. A valuation of a matroid $(V, \mathcal{B})$, defined on $V$ in terms of the basis family $\mathcal{B}$, is a function $\omega: \mathcal{B} \rightarrow R$ which enjoys the exchange property:

(MV) For $X, Y \in \mathcal{B}$ and $u \in X-Y$ there exists $v \in Y-X$ such that $X-u+v \in \mathcal{B}$, $Y+u-v \in \mathcal{B}$ and

$$
\omega(X)+\omega(Y) \leq \omega(X-u+v)+\omega(Y+u-v) .
$$

A matroid equipped with a valuation is called valuated matroid. By considering the case of $\omega(X)=0$ for all $X \in \mathcal{B}$ we see that (MV) is a quantitative generalization of the simultaneous exchange property of a matroid.

It has turned out recently that the valuated matroids afford a nice combinatorial framework to which the optimization algorithms for matroids can be generalized. Variants of greedy algorithms work for maximizing a matroid valuation, as has been shown by Dress-Wenzel [6] as well as by Dress-Terhalle [3, 4, 5] and Murota [24]. The weighted matroid intersection problem has been extended by Murota [25], [26] using valuations to the "valuated matroid intersection problem." The optimality criteria and algorithms for the weighted matroid intersection problem have been generalized for the valuated matroid intersection problem.

In this paper we consider a common generalization of the submodular flow problem and the valuated matroid intersection problem. In addition to the arc cost $\sum_{a \in A} \gamma(a) \varphi(a)$ in the submodular flow problem (P0), we introduce a new term $-\omega(\partial \varphi)$ to express the cost of the boundary $\partial \varphi$ in the objective function, where $\omega: B \rightarrow R$ is a nonseparable function in general. Namely, we consider the following.

[Generalized submodular flow problem $(\mathrm{P})$ ]

Minimize

$$
\Gamma(\varphi)=\sum_{a \in A} \gamma(a) \varphi(a)-\omega(\partial \varphi)
$$


subject to

$$
\begin{aligned}
& \underline{c}(a) \leq \varphi(a) \leq \bar{c}(a) \quad(a \in A), \\
& \partial \varphi \in B .
\end{aligned}
$$

The major assumption in this paper is that the function $\omega: B \rightarrow R$ should satisfy the following variant of Steinitz's exchange property:

$\left(\mathbf{E X C}_{0}\right)$ For $x, y \in B$ and $u \in \operatorname{supp}^{+}(x-y)$ there exists $v \in \operatorname{supp}^{-}(x-y)$ such that $x-\chi_{u}+\chi_{v} \in B, y+\chi_{u}-\chi_{v} \in B$ and

$$
\omega(x)+\omega(y) \leq \omega\left(x-\chi_{u}+\chi_{v}\right)+\omega\left(y+\chi_{u}-\chi_{v}\right),
$$

where $\operatorname{supp}^{+}(x-y)=\{u \in V \mid x(u)>y(u)\}, \operatorname{supp}^{-}(x-y)=\{v \in V \mid x(v)<$ $y(v)\}$, and $\chi_{u}$ and $\chi_{v}$ denote the characteristic vectors of $u$ and $v$, respectively. Let us say that such a function $\omega$ is M-concave ${ }^{2}$. Obviously, $\left(\mathrm{EXC}_{0}\right)$ is a generalization of the exchange property $(\mathrm{MV})$ of a matroid valuation in the sense that $\left(\mathrm{EXC}_{0}\right)$ reduces to (MV) if $B \subseteq\{0,1\}^{V}$.

The M-concavity is inherent in the ordinary submodular flow problem (P0) itself. Define $B_{0} \subseteq \mathbf{Z}^{V}$ by

$$
B_{0}=\{\partial \varphi \mid \underline{c}(a) \leq \varphi(a) \leq \bar{c}(a)(a \in A)\}
$$

and $\omega_{0}: B_{0} \rightarrow R$ by

$$
\omega_{0}(x)=-\min \left\{\Gamma_{0}(\varphi) \mid \partial \varphi=x, \underline{c}(a) \leq \varphi(a) \leq \bar{c}(a)(a \in A)\right\} .
$$

Then (P0) can be written as

$$
\text { Maximize } \omega_{0}(x) \text { subject to } x \in B_{0} \cap B
$$

where $x=\partial \varphi$. In this formulation $B_{0}$ is a base polyhedron (a well-known fact) and $\omega_{0}$ is M-concave as will be shown in Example 2.3.

The generalized submodular flow problem $(\mathrm{P})$ can be recast into a number of different forms through simple transformations. Firstly, a separable convex arc cost can be described within this framework. That is, it will be shown that $(\mathrm{P})$ is as general as the problem with an objective function of the form:

$$
\Gamma_{2}(\varphi)=\sum_{a \in A} f_{a}(\varphi(a))-\omega(\partial \varphi)
$$

\footnotetext{
${ }^{2}$ The name "M-concavity" is intended to mean a concavity related to Matroid. The relationship of the exchange axiom $\left(\mathrm{EXC}_{0}\right)$ and the concavity in the ordinary sense is investigated in [30], [31].
} 
where $f_{a}$, for each $a \in A$, is a convex function and $\omega$ is an M-concave function. This includes, as a special case with $\omega \equiv 0$, the submodular flow problem with a separable convex cost function considered by Fujishige [17, Chapter 12]. The latter problem contains, as a further special case, the integer minimum-cost flow problem with a separable convex objective function investigated by Minoux [23].

Another equivalent form of $(\mathrm{P})$ is obtained through the M-concave function $\omega_{0}$ above. Namely, the problem $(\mathrm{P})$ can be reformulated into the "intersection problem":

$$
\text { Maximize } \omega_{0}(x)+\omega(x) \text { subject to } x \in B_{0} \cap B,
$$

where $x=\partial \varphi$. Since $\omega_{0}(x)$ can be computed efficiently by means of the wellestablished network-flow algorithms, we may regard this problem as being equivalent to $(\mathrm{P})$. Conversely, for two M-concave functions $\omega_{0}$ and $\omega$ in general, the problem (1.6) can be described easily in the form of (P). Notice also that the introduction of the new term $\omega(\partial \varphi)$ brings (1.4) into a more symmetric form (1.6).

In this way we may say that the M-concavity $\left(\mathrm{EXC}_{0}\right)$ is quite natural in the context of the submodular flow problem.

In the present paper we establish two forms of optimality criteria for the generalized submodular flow problem (P). The first criterion (Theorem 3.1) is in terms of potentials (or dual variables) associated with vertices and the second (Theorem $3.2)$ in terms of negative cycles in an auxiliary graph. These criteria are natural extensions of the well-known results for the ordinary submodular flow problem (P0) with a linear cost function (Frank [12], Frank-Tardos [14], Fujishige [15], Zimmermann [36]) and with a separable convex cost function (Fujishige [17]), as well as the recent result of the author (Murota [25]) for the valuated matroid intersection problem.

In the following section we first investigate the M-concavity itself, independently of the submodular flow problem. We identify three classes of M-concave functions; affine functions on the base polyhedron, separable concave functions on the base polyhedron, and the minimum cost of a flow that meets a specified boundary requirement (as $\omega_{0}$ above). As already mentioned, these examples demonstrate that the M-concavity $\left(\mathrm{EXC}_{0}\right)$ is a natural property in relation to the submodular flow problem. Then we go on to investigate the fundamental properties implied by $\left(\mathrm{EXC}_{0}\right)$. These are mostly straightforward extensions of the results for matroid valuations due to Dress-Wenzel [7] and Murota [25]. With these fundamental properties we state in Section 3 the optimality criteria for the generalized submodular flow problem (P), while postponing the proofs to Section 5. As a corollary a discrete separation theorem for a pair of M-concave/convex functions is derived in Section 4. Appendix gives the reduction of a separable convex arc cost to a boundary cost with the exchange property $\left(\mathrm{EXC}_{0}\right)$, and the proof for the equivalence between $\left(\mathrm{EXC}_{0}\right)$ and an alternative (seemingly weaker) exchange 
property.

\section{M-concave Functions}

\subsection{Definitions}

Let $V$ be a finite set, and $R$ be a totally ordered additive group (typically $R=\mathbf{R}$ (reals), $\mathbf{Q}$ (rationals), or $\mathbf{Z}$ (integers)). For $x=(x(v) \mid v \in V) \in \mathbf{Z}^{V}, y=(y(v) \mid$ $v \in V) \in R^{V}$ we define

$$
\begin{gathered}
\operatorname{supp}^{+}(x)=\{v \in V \mid x(v)>0\}, \quad \operatorname{supp}^{-}(x)=\{v \in V \mid x(v)<0\}, \\
x(V)=\sum\{x(v) \mid v \in V\}, \quad\|x\|=\sum\{|x(v)| \mid v \in V\}, \\
\langle x, y\rangle=\langle y, x\rangle=\sum\{x(v) y(v) \mid v \in V\} .
\end{gathered}
$$

For $u \in V$ we denote by $\chi_{u}$ its characteristic vector, i.e., $\chi_{u}=\left(\chi_{u}(v) \mid v \in V\right) \in \mathbf{Z}^{V}$ with $\chi_{u}(v)=1$ if $v=u$ and $\chi_{u}(v)=0$ otherwise.

We consider a function $\omega: \mathbf{Z}^{V} \rightarrow R \cup\{-\infty\}$ that satisfies the following variant of Steinitz's exchange property:

(EXC) For $x, y \in \mathbf{Z}^{V}$ with $\omega(x) \neq-\infty$ and $\omega(y) \neq-\infty$, and for $u \in \operatorname{supp}^{+}(x-y)$, there exists $v \in \operatorname{supp}^{-}(x-y)$ such that

$$
\omega(x)+\omega(y) \leq \omega\left(x-\chi_{u}+\chi_{v}\right)+\omega\left(y+\chi_{u}-\chi_{v}\right) .
$$

We exclude the case where $\omega=-\infty$ identically, namely, we assume that

$$
B \equiv\left\{x \in \mathbf{Z}^{V} \mid \omega(x) \neq-\infty\right\} \neq \emptyset .
$$

Such a function $\omega: \mathbf{Z}^{V} \rightarrow R \cup\{-\infty\}$ will be called M-concave, whereas $-\omega$ is M-convex.

To avoid terms of $-\infty$ in the inequality (2.1) we may alternatively say:

$\left(\mathbf{E X C}_{0}\right)$ For $x, y \in B$ and $u \in \operatorname{supp}^{+}(x-y)$ there exists $v \in \operatorname{supp}^{-}(x-y)$ such that $x-\chi_{u}+\chi_{v} \in B, y+\chi_{u}-\chi_{v} \in B$ and

$$
\omega(x)+\omega(y) \leq \omega\left(x-\chi_{u}+\chi_{v}\right)+\omega\left(y+\chi_{u}-\chi_{v}\right) .
$$

The M-concavity can be regarded as a quantitative extension of the concept of the base polyhedron of a submodular system [17], just as the matroid valuation is a quantitative extension of the matroid. In fact, it follows from $\left(\mathrm{EXC}_{0}\right)$ that for $x, y \in B$ and $u \in \operatorname{supp}^{+}(x-y)$, there exists $v \in \operatorname{supp}^{-}(x-y)$ such that $x-\chi_{u}+\chi_{v} \in B, y+\chi_{u}-\chi_{v} \in B$. This simultaneous exchange property is 
equivalent to the condition that $B$ is (the integral points of) the base polyhedron of an integral submodular system, since the simultaneous exchange property is equivalent (cf. Lemma 2.1 below) to a seemingly weaker property: for $x, y \in B$ and $u \in \operatorname{supp}^{+}(x-y)$, there exists $v \in \operatorname{supp}^{-}(x-y)$ such that $x-\chi_{u}+\chi_{v} \in B$, which is known ${ }^{3}$ to characterizes the base polyhedron of a submodular system.

The following lemma may be thought of as a folklore, in which (B2) is well known to be satisfied if $B$ is (the integral points of) the base polyhedron of an integral submodular system [17].

Lemma 2.1 For $B \subseteq \mathbf{Z}^{V}$ the following three conditions, (B1), (B2) and (B3), are equivalent.

(B1) For distinct $x, y \in B$, there exist $u \in \operatorname{supp}^{+}(x-y)$ and $v \in \operatorname{supp}^{-}(x-y)$ such that $x-\chi_{u}+\chi_{v} \in B, y+\chi_{u}-\chi_{v} \in B$.

(B2) For distinct $x, y \in B$ and for $u \in \operatorname{supp}^{+}(x-y)$, there exists $v \in \operatorname{supp}^{-}(x-y)$ such that $x-\chi_{u}+\chi_{v} \in B$.

(B3) For distinct $x, y \in B$ and for $u \in \operatorname{supp}^{+}(x-y)$, there exists $v \in \operatorname{supp}^{-}(x-y)$ such that $x-\chi_{u}+\chi_{v} \in B, y+\chi_{u}-\chi_{v} \in B$.

(Proof) (B3) $\Rightarrow(\mathrm{B} 2)$ and (B3) $\Rightarrow(\mathrm{B} 1)$ are obvious. (B1) $\Rightarrow$ (B3) is a special case of Theorem 2.2 below, and $(\mathrm{B} 2) \Rightarrow(\mathrm{B} 3)$ is shown in Appendix A.3. It is remarked that $(\mathrm{B} 1) \Leftrightarrow(\mathrm{B} 2)$ is explicit in Tomizawa [35].

We mention here that the exchange property (EXC) is equivalent to the following seemingly weaker property $\left(\mathrm{EXC}_{\mathrm{w}}\right)$, though we do not make use of this fact later in this paper. Note that $\left(\mathrm{EXC}_{\mathrm{w}}\right)$ corresponds to $(\mathrm{B} 1)$ in Lemma 2.1.

Theorem 2.2 For $\omega: \mathbf{Z}^{V} \rightarrow R \cup\{-\infty\}$ with (2.2), (EXC) is equivalent to

$\left(\mathbf{E X C}_{\mathrm{w}}\right)$ For distinct $x, y \in \mathbf{Z}^{V}$, there exist $u \in \operatorname{supp}^{+}(x-y)$ and $v \in \operatorname{supp}^{-}(x-y)$ such that

$$
\omega(x)+\omega(y) \leq \omega\left(x-\chi_{u}+\chi_{v}\right)+\omega\left(y+\chi_{u}-\chi_{v}\right) .
$$

(Proof) See Appendix A.2.

\footnotetext{
${ }^{3}$ This is a folk theorem, according to private communications from W. Cunningham and S. Fujishige.
} 


\subsection{Examples of M-concave functions}

We show three natural classes of M-concave functions that, by definition, satisfy the exchange axiom (EXC). See also Dress-Wenzel [7] and Murota [25], [29] for instances of matroid valuations.

Example 2.1 (Affine function) Let $B \subseteq \mathbf{Z}^{V}$ be (the integral points of) the base polyhedron of an integral submodular system [17]. For $\eta: V \rightarrow R$ and $\alpha \in R$, the function $\omega: \mathbf{Z}^{V} \rightarrow R \cup\{-\infty\}$ defined by

$$
\omega(x)= \begin{cases}\alpha+\langle\eta, x\rangle & (x \in B) \\ -\infty & (x \notin B)\end{cases}
$$

satisfies the exchange property (EXC) with equality in (2.1). This is an immediate consequence of the symmetric exchange property (B3) in Lemma 2.1, since as is well known, $B$ satisfies (B2) there.

Example 2.2 (Separable concave function) Let $B \subseteq \mathbf{Z}^{V}$ be (the integral points of) the base polyhedron of an integral submodular system [17]. We call $g: \mathbf{Z} \rightarrow R$ concave if its piecewise linear extension $\widehat{g}: \mathbf{R} \rightarrow R$ is a concave function. For a family of concave functions $g_{v}: \mathbf{Z} \rightarrow R$ indexed by $v \in V$, the (separable concave) function $\omega: \mathbf{Z}^{V} \rightarrow R \cup\{-\infty\}$ defined by

$$
\omega(x)= \begin{cases}\sum\left\{g_{v}(x(v)) \mid v \in V\right\} & (x \in B) \\ -\infty & (x \notin B)\end{cases}
$$

satisfies the exchange property (EXC).

To see this, take $x, y \in B$ and $u \in \operatorname{supp}^{+}(x-y)$. By (B3) in Lemma 2.1 there exists $v \in \operatorname{supp}^{-}(x-y)$ such that $x^{\prime} \equiv x-\chi_{u}+\chi_{v} \in B, y^{\prime} \equiv y+\chi_{u}-\chi_{v} \in B$. Then we have

$$
\begin{aligned}
\omega\left(x^{\prime}\right)+\omega\left(y^{\prime}\right)= & {\left[g_{u}(x(u)-1)+g_{u}(y(u)+1)\right]+\left[g_{v}(x(v)+1)+g_{v}(y(v)-1)\right] } \\
& +\sum_{w \neq u, v}\left[g_{w}(x(w))+g_{w}(y(w))\right] .
\end{aligned}
$$

By the concavity of $g_{u}$ and the relations $y(u) \leq x(u)-1 \leq x(u)$ and $y(u) \leq$ $y(u)+1 \leq x(u)$, we see that

$$
g_{u}(x(u)-1)+g_{u}(y(u)+1) \geq g_{u}(x(u))+g_{u}(y(u)) .
$$

Similarly we have

$$
g_{v}(x(v)+1)+g_{v}(y(v)-1) \geq g_{v}(x(v))+g_{v}(y(v)) .
$$

Hence we obtain $\omega\left(x^{\prime}\right)+\omega\left(y^{\prime}\right) \geq \omega(x)+\omega(y)$. 
Example 2.3 (Min-cost flow) Let $G=(V, A)$ be a graph with a vertex set $V$ and an $\operatorname{arc}$ set $A$. Assume further that we are given an upper capacity function $\bar{c}: A \rightarrow \mathbf{Z} \cup\{+\infty\}$, a lower capacity function $\underline{c}: A \rightarrow \mathbf{Z} \cup\{-\infty\}$, and a cost function $\gamma: A \rightarrow R$. It is assumed that each directed cycle has a nonnegative cost with respect to $\gamma$. A feasible (integral) flow $\varphi$ is a function $\varphi: A \rightarrow \mathbf{Z}$ such that $\underline{c}(a) \leq \varphi(a) \leq \bar{c}(a)$ for each $a \in A$. Its boundary $\partial \varphi: V \rightarrow \mathbf{Z}$ is defined by

$$
\partial \varphi(v)=\sum\left\{\varphi(a) \mid a \in \delta^{+} v\right\}-\sum\left\{\varphi(a) \mid a \in \delta^{-} v\right\}
$$

where $\delta^{+} v$ and $\delta^{-} v$ denote the sets of the out-going and in-coming arcs incident to $v$, respectively. Then the function $\omega: \mathbf{Z}^{V} \rightarrow R \cup\{-\infty\}$ defined by

$$
\begin{aligned}
\omega(x) & =-\min \left\{\Gamma_{0}(\varphi) \mid \varphi: \text { feasible flow with } \partial \varphi=x\right\}, \\
\Gamma_{0}(\varphi) & =\langle\gamma, \varphi\rangle=\sum\{\gamma(a) \varphi(a) \mid a \in A\}
\end{aligned}
$$

satisfies the exchange property (EXC), as explained below. By convention we put $\omega(x)=-\infty$ if there is no feasible $\varphi$ with $\partial \varphi=x$. Note that $\partial \varphi(V)=0$ and hence $x(V)=0$ if $\omega(x) \neq-\infty$. In general this construction yields a nonseparable function $\omega$ (see [25, Example 3.3] for a concrete instance).

To see (EXC), consider $x, y \in \mathbf{Z}^{V}$ with $x(V)=y(V)=0, \omega(x) \neq-\infty$, $\omega(y) \neq-\infty$, and $u \in \operatorname{supp}^{+}(x-y)$. Take feasible $\varphi_{x}, \varphi_{y}$ such that $\omega(x)=-\gamma\left(\varphi_{x}\right)$, $\omega(y)=-\gamma\left(\varphi_{y}\right), \partial \varphi_{x}=x, \partial \varphi_{y}=y$. By a standard augmenting-path argument we see that there is $\pi: A \rightarrow\{0, \pm 1\}$ such that $\operatorname{supp}^{+}(\pi) \subseteq \operatorname{supp}^{+}(x-y), \operatorname{supp}^{-}(\pi) \subseteq$ $\operatorname{supp}^{-}(x-y)$ and that $\partial \pi=\chi_{u}-\chi_{v}$ for some $v \in \operatorname{supp}^{-}(x-y)$. Then we have $\partial\left(\varphi_{x}-\pi\right)=x-\chi_{u}+\chi_{v}, \partial\left(\varphi_{y}+\pi\right)=y+\chi_{u}-\chi_{v}$ and

$$
\begin{aligned}
& \omega(x)+\omega(y)=-\left\langle\gamma, \varphi_{x}\right\rangle-\left\langle\gamma, \varphi_{y}\right\rangle=-\left\langle\gamma, \varphi_{x}-\pi\right\rangle-\left\langle\gamma, \varphi_{y}+\pi\right\rangle \\
& \leq-\min \left\{\langle\gamma, \varphi\rangle \mid \partial \varphi=x-\chi_{u}+\chi_{v}\right\}-\min \left\{\langle\gamma, \varphi\rangle \mid \partial \varphi=y+\chi_{u}-\chi_{v}\right\} \\
& =\omega\left(x-\chi_{u}+\chi_{v}\right)+\omega\left(y+\chi_{u}-\chi_{v}\right) .
\end{aligned}
$$

This construction remains valid in the more general case where the cost is given by a separable convex function. We call $f: \mathbf{Z} \rightarrow R$ convex if its piecewise linear extension $\widehat{f}: \mathbf{R} \rightarrow R$ is a convex function. For a family of convex functions $f_{a}: \mathbf{Z} \rightarrow R$ indexed by $a \in A$, the function $\omega: \mathbf{Z}^{V} \rightarrow R \cup\{-\infty\}$ defined by

$$
\begin{aligned}
\omega(x) & =-\min \left\{\Gamma_{1}(\varphi) \mid \varphi: \text { feasible flow with } \partial \varphi=x\right\}, \\
\Gamma_{1}(\varphi) & =\sum\left\{f_{a}(\varphi(a)) \mid a \in A\right\},
\end{aligned}
$$

satisfies the exchange property (EXC) under the assumption that the minimum of $\Gamma_{1}(\varphi)$ over $\varphi$ with $\partial \varphi=x$ is bounded from below for all $x \in B$. This can be shown by the above argument, combined with the following relation:

$$
\Gamma_{1}\left(\varphi_{x}-\pi\right)+\Gamma_{1}\left(\varphi_{y}+\pi\right)=\sum_{a: \pi(a)=1}\left[f_{a}\left(\varphi_{x}(a)-1\right)+f_{a}\left(\varphi_{y}(a)+1\right)\right]
$$




$$
\begin{aligned}
& +\sum_{a: \pi(a)=-1}\left[f_{a}\left(\varphi_{x}(a)+1\right)+f_{a}\left(\varphi_{y}(a)-1\right)\right] \\
& +\sum_{a: \pi(a)=0}\left[f_{a}\left(\varphi_{x}(a)\right)+f_{a}\left(\varphi_{y}(a)\right)\right] \\
& \leq \Gamma_{1}\left(\varphi_{x}\right)+\Gamma_{1}\left(\varphi_{y}\right),
\end{aligned}
$$

which follows from

$$
\begin{aligned}
& f_{a}\left(\varphi_{x}(a)-1\right)+f_{a}\left(\varphi_{y}(a)+1\right) \leq f_{a}\left(\varphi_{x}(a)\right)+f_{a}\left(\varphi_{y}(a)\right) \quad \text { if } \varphi_{x}(a)>\varphi_{y}(a), \\
& f_{a}\left(\varphi_{x}(a)+1\right)+f_{a}\left(\varphi_{y}(a)-1\right) \leq f_{a}\left(\varphi_{x}(a)\right)+f_{a}\left(\varphi_{y}(a)\right) \quad \text { if } \varphi_{x}(a)<\varphi_{y}(a) .
\end{aligned}
$$

Remark 2.1 In connection to the construction in Example 2.2 it is mentioned that a general concave function on $\mathbf{R}^{V}$ (or on a base polyhedron over $\mathbf{R}$ ) does not satisfy (EXC) when restricted to $\mathbf{Z}^{V}$. For example, let $V=\left\{v_{1}, v_{2}, v_{3}, v_{4}\right\}$,

$$
b_{1}=\chi_{v_{1}}+\chi_{v_{2}}, \quad b_{2}=\chi_{v_{3}}+\chi_{v_{4}}, \quad b_{3}=\chi_{v_{1}}+\chi_{v_{3}}, \quad b_{4}=\chi_{v_{2}}+\chi_{v_{4}},
$$

$B=\left\{b_{1}, b_{2}, b_{3}, b_{4}\right\} \subseteq \mathbf{Z}^{V}$, and $\bar{B} \subseteq \mathbf{R}^{V}$ be the convex hull of $B$. That is, $\bar{B}$ is the base polytope of the matroid on $V$ with the basis family $\left\{\left\{v_{1}, v_{2}\right\},\left\{v_{3}, v_{4}\right\},\left\{v_{1}, v_{3}\right\}\right.$, $\left.\left\{v_{2}, v_{4}\right\}\right\}$. A point $x$ in $\bar{B}$ can be expressed as

$$
x=\lambda_{1} b_{1}+\lambda_{2} b_{2}+\left(1-\lambda_{1}-\lambda_{2}\right) b
$$

with $0 \leq \lambda_{i} \leq 1(i=1,2), \lambda_{1}+\lambda_{2} \leq 1$ and $b=b_{3}$ or $b_{4}$. According to this expression define $g: \mathbf{R}^{V} \rightarrow \mathbf{R} \cup\{-\infty\}$ by

$$
g(x)= \begin{cases}\lambda_{1}+\lambda_{2} & (x \in \bar{B}) \\ -\infty & (x \notin \bar{B})\end{cases}
$$

which is well-defined to be a piecewise linear concave function. The restriction of $g$ to $\mathbf{Z}^{V}$, given by

$$
g\left(b_{1}\right)=g\left(b_{2}\right)=1, \quad g\left(b_{3}\right)=g\left(b_{4}\right)=0, \quad g(x)=-\infty \quad(x \notin B),
$$

does not satisfy (EXC). (The relationship of the M-concavity and the ordinary concavity is investigated in [30], [31].) 


\subsection{Fundamental properties}

We assume $\omega: \mathbf{Z}^{V} \rightarrow R \cup\{-\infty\}$ is M-concave, satisfying (EXC), and that

$$
B=\left\{x \in \mathbf{Z}^{V} \mid \omega(x) \neq-\infty\right\}
$$

is a nonempty subset of $\left\{x \in \mathbf{Z}^{V} \mid x(V)=0\right\}$. We introduce the notation

$$
\omega(x, u, v)=\omega\left(x-\chi_{u}+\chi_{v}\right)-\omega(x) \quad(x \in B ; u, v \in V) .
$$

As the definition shows, $\omega(x, u, v)$ represents the information about the local behavior of $\omega$ in the neighborhood of $x$. Note also that the inequality (2.1) for (EXC) can be rewritten to

$$
\omega(x, u, v)+\omega(y, v, u) \geq 0 .
$$

The following fact is fundamental, showing the local optimality implies the global optimality.

Theorem 2.3 Let $x \in B$. Then $\omega(x) \geq \omega(y)(y \in B)$ if and only if

$$
\omega(x, u, v) \leq 0 \quad(u, v \in V)
$$

(Proof) The necessity is obvious. We prove the sufficiency by induction on $\|x-y\|$. The assumption implies $\omega(x) \geq \omega(y)$ for $y \in B$ with $\|x-y\|=2$. Suppose $\|x-y\| \geq 4$. By (EXC) there exists $x^{\prime}, y^{\prime} \in B$ such that $\left\|x-x^{\prime}\right\|=\left\|y-y^{\prime}\right\|=2$, $\left\|x-y^{\prime}\right\|=\|x-y\|-2$, and

$$
\omega(x)+\omega(y) \leq \omega\left(x^{\prime}\right)+\omega\left(y^{\prime}\right) .
$$

Here we have $\omega\left(x^{\prime}\right) \leq \omega(x)$ by the assumption and $\omega\left(y^{\prime}\right) \leq \omega(x)$ by the induction hypothesis. Hence we obtain $\omega(y) \leq \omega(x)$. (An alternative proof is given later in Remark 2.5.)

Remark 2.2 The above theorem is a straightforward extension of the result of Dress-Wenzel [6], [7] for a matroid valuation.

As a refinement of Theorem 2.3 we will derive an upper estimate of $\omega(y)-\omega(x)$ in terms of the local information $\omega(x, u, v)(u, v \in V)$ in the neighborhood of $x$. For $x \in B$ and $y \in \mathbf{Z}^{V}$ we consider a bipartite graph $G(x, y)=\left(V^{+}, V^{-} ; \widehat{A}\right)$, where $\left(V^{+}, V^{-}\right)=\left(\operatorname{supp}^{+}(x-y), \operatorname{supp}^{-}(x-y)\right)$ is the vertex bipartition and

$$
\widehat{A}=\left\{(u, v) \mid u \in V^{+}, v \in V^{-}, x-\chi_{u}+\chi_{v} \in B\right\}
$$


is the arc set. Each $\operatorname{arc}(u, v)$ is associated with "arc weight" $\omega(x, u, v)$ of $(2.4)$. We define

$$
\begin{aligned}
\widehat{\omega}(x, y)=\max & \left\{\sum_{(u, v) \in \widehat{A}} \omega(x, u, v) \lambda(u, v) \mid\right. \\
\lambda(u, v) \geq 0 \quad((u, v) \in \widehat{A}), & \left(u \in V^{+}\right), \\
& \sum_{v:(u, v) \in \widehat{A}} \lambda(u, v)=x(u)-y(u) \quad \\
& \left.\sum_{u:(u, v) \in \widehat{A}} \lambda(u, v)=y(v)-x(v) \quad\left(v \in V^{-}\right)\right\} .
\end{aligned}
$$

By convention, the maximum taken over an empty family is understood to be $-\infty$. It is not difficult to recognize this problem as a transportation problem [32], in which the cost of $\operatorname{arc}(u, v)$ is given by $-\omega(x, u, v)$. The following lemma shows that $\widehat{\omega}(x, y)$ serves as an upper bound on $\omega(y)-\omega(x)$.

Lemma 2.4 ("upper-bound lemma") For $x, y \in B$ we have

$$
\omega(y) \leq \omega(x)+\widehat{\omega}(x, y) .
$$

(Proof) For any $u_{1} \in \operatorname{supp}^{+}(x-y)$ there exists $v_{1} \in \operatorname{supp}^{-}(x-y)$ with

$$
\omega(x)+\omega(y) \leq \omega\left(x-\chi_{u_{1}}+\chi_{v_{1}}\right)+\omega\left(y+\chi_{u_{1}}-\chi_{v_{1}}\right),
$$

which can be rewritten as

$$
\omega(y) \leq \omega\left(x, u_{1}, v_{1}\right)+\omega\left(y_{2}\right)
$$

with $y_{2}=y+\chi_{u_{1}}-\chi_{v_{1}}$. By the same argument applied to $\left(x, y_{2}\right)$ we obtain

$$
\omega\left(y_{2}\right) \leq \omega\left(x, u_{2}, v_{2}\right)+\omega\left(y_{3}\right)
$$

for some $u_{2} \in \operatorname{supp}^{+}\left(x-y_{2}\right)$ and $v_{2} \in \operatorname{supp}^{-}\left(x-y_{2}\right)$, where $y_{3}=y_{2}+\chi_{u_{2}}-\chi_{v_{2}}=$ $y-\chi_{u_{1}}-\chi_{u_{2}}+\chi_{v_{1}}+\chi_{v_{2}}$. Hence

$$
\omega(y) \leq \omega\left(y_{3}\right)+\sum_{i=1}^{2} \omega\left(x, u_{i}, v_{i}\right) .
$$

Repeating this process we arrive at

$$
\omega(y) \leq \omega(x)+\sum_{i=1}^{m} \omega\left(x, u_{i}, v_{i}\right) \leq \omega(x)+\widehat{\omega}(x, y)
$$

where $m=\|x-y\| / 2, y=x-\sum_{i=1}^{m}\left(\chi_{u_{i}}-\chi_{v_{i}}\right)$. 
Remark 2.3 The "upper-bound lemma" (Lemma 2.4) is an extension of a similar lemma of the same name (Lemma 3.4 of Murota [25]) for a valuated matroid.

Remark 2.4 The bipartite graph $G(x, y)$ agrees with the exchangeability graph employed in the submodular function theory [17]. Moreover, the "upper-bound lemma" (Lemma 2.4) can be regarded as a quantitative extension of the wellknown fact for a submodular system (cf. [17, Theorem 3.28]), which reads in our notation that $\widehat{\omega}(x, y) \neq-\infty$ for $x, y \in B$. In fact, this is implied by (2.8) with $\omega(y)$ and $\omega(x)$ finite.

Remark 2.5 The "upper-bound lemma" (Lemma 2.4) can be considered a refinement of Theorem 2.3 since it gives an easy alternative proof for (the sufficiency of) the optimality condition in Theorem 2.3. Take any $y \in B$ and consider $G(x, y)$. The condition (2.6) is equivalent to all the arcs having nonpositive weights. Hence $\widehat{\omega}(x, y) \leq 0$, which implies $\omega(y) \leq \omega(x)$ by Lemma 2.4 .

It would be natural to ask for a condition under which the inequality in (2.8) reduces to an equality. For $(x, y)$ with $x \in B, y \in \mathbf{Z}^{V}$ and $|x(v)-y(v)| \leq 1$ $(v \in V)$, we consider

\section{[Unique-Max Condition]}

There exists exactly one maximum-weight perfect matching in $G(x, y)$.

Then we have the following lemma, which is an extension of the well-known "noshortcut lemma" for matroids [1, Lemma 3.7], [11, Lemma 2], is most crucial in the proof of the optimality criteria in Section 5.

Lemma 2.5 ("unique-max lemma" [25, Lemma 3.8]) Let $(x, y)$ be such that $x \in B, y \in \mathbf{Z}^{V}$ and $|x(v)-y(v)| \leq 1(v \in V)$. If $(x, y)$ satisfies the unique-max condition, then $y \in B$ and

$$
\omega(y)=\omega(x)+\widehat{\omega}(x, y) .
$$

(Proof) For $z \in \mathcal{I} \equiv\left\{z \in \mathbf{Z}^{V} \mid \min (x(v), y(v)) \leq z(v) \leq \max (x(v), y(v))(v \in V)\right\}$ put $S(z)=\{v \in V \mid z(v)>\min (x(v), y(v))\}$. Define $\omega^{\prime}: 2^{V} \rightarrow R \cup\{-\infty\}$ by

$$
\omega^{\prime}(Z)= \begin{cases}\omega(z) & (\exists z \in \mathcal{I}: Z=S(z)) \\ -\infty & \text { (otherwise) }\end{cases}
$$

Then $\left(V, \omega^{\prime}\right)$ is a valuated matroid, to which Lemma 3.8 of [25] applies.

It is noted that the unique-max condition can be expressed in terms of "potential" or "dual variable" as follows. This shows at the same time how to check the unique-max condition in polynomial time. 
Lemma 2.6 ([25, Lemma 3.5]) Let $(x, y)$ be such that $x \in B, y \in \mathbf{Z}^{V}$ and $|x(v)-y(v)| \leq 1(v \in V)$.

(1) $G(x, y)$ has a perfect matching if and only if there exist $\widehat{p}: V^{+} \cup V^{-} \rightarrow R$ and indexings of the elements of $V^{+}$and $V^{-}$, say $V^{+}=\left\{u_{1}, \cdots, u_{m}\right\}, V^{-}=$ $\left\{v_{1}, \cdots, v_{m}\right\}$, such that, for any $\left(u_{i}, v_{j}\right) \in \widehat{A}$,

$$
\omega\left(x, u_{i}, v_{j}\right)-\widehat{p}\left(u_{i}\right)+\widehat{p}\left(v_{j}\right) \begin{cases}=0 & (1 \leq i=j \leq m) \\ \leq 0 & (1 \leq i, j \leq m)\end{cases}
$$

Furthermore, the relation (2.12) implies

$$
\widehat{\omega}(x, y)=\sum_{i=1}^{m}\left(\widehat{p}\left(u_{i}\right)-\widehat{p}\left(v_{i}\right)\right) .
$$

(2) The pair $(x, y)$ satisfies the unique-max condition if and only if there exist $\widehat{p}: V^{+} \cup V^{-} \rightarrow R$ and indexings of the elements of $V^{+}$and $V^{-}$, say $V^{+}=$ $\left\{u_{1}, \cdots, u_{m}\right\}, V^{-}=\left\{v_{1}, \cdots, v_{m}\right\}$, such that, for any $\left(u_{i}, v_{j}\right) \in \widehat{A}$,

$$
\omega\left(x, u_{i}, v_{j}\right)-\widehat{p}\left(u_{i}\right)+\widehat{p}\left(v_{j}\right) \begin{cases}=0 & (1 \leq i=j \leq m) \\ \leq 0 & (1 \leq j<i \leq m) \\ <0 & (1 \leq i<j \leq m)\end{cases}
$$

(Proof) This is immediate from the duality (complementarity) in the weighted bipartite matching problem, being independent of the M-concavity of $\omega$.

\section{Optimality Criteria}

We give two optimality criteria for the problem $(\mathrm{P})$, as well as a necessary and sufficient condition for the existence of an optimal flow. Both of these optimality criteria (Theorems 3.1 and 3.2) are natural extensions of the corresponding results for the submodular flow problem (P0) (see Cunningham-Frank [2], Frank [12], Frank-Tardos [14], Fujishige [15] [17], Zimmermann [36]). Through the transformation of the arc cost to the boundary cost explained in Appendix, they also imply the optimality criteria of Fujishige [17, Chapter 12] for the submodular flow problem with a separable convex cost function.

Let us recall that the problem $(\mathrm{P})$ is defined on $G=(V, A)$ with an upper capacity function $\bar{c}: A \rightarrow \mathbf{Z} \cup\{+\infty\}$, a lower capacity function $\underline{c}: A \rightarrow \mathbf{Z} \cup\{-\infty\}$, and cost functions $\gamma: A \rightarrow R$ and $\omega: \mathbf{Z}^{V} \rightarrow R \cup\{-\infty\}$, where $R$ is a totally ordered additive group (e.g., $R=\mathbf{R}, \mathbf{Q}$, or $\mathbf{Z}$ ) and $\omega$ is an M-concave function. A function $\varphi: A \rightarrow \mathbf{Z}$ is a feasible flow if $\underline{c}(a) \leq \varphi(a) \leq \bar{c}(a)$ for each $a \in A$ and $\partial \varphi \in B \equiv\left\{x \in \mathbf{Z}^{V} \mid \omega(x) \neq-\infty\right\}$. 
The first optimality criterion refers to a "potential" function. For $p: V \rightarrow R$ we define $\gamma_{p}: A \rightarrow R$ by

$$
\gamma_{p}(a)=\gamma(a)+p\left(\partial^{+} a\right)-p\left(\partial^{-} a\right) \quad(a \in A) .
$$

For $p: V \rightarrow R$ we define $\omega[p]: \mathbf{Z}^{V} \rightarrow R \cup\{-\infty\}$ by

$$
\omega[p](x)=\omega(x)+\langle p, x\rangle .
$$

It is emphasized that in the case of $R=\mathbf{Z}$ the integrality of $p$ is an essential ingredient of the assertion of the theorem below.

Theorem 3.1 (1) A feasible flow $\varphi: A \rightarrow \mathbf{Z}$ is optimal for $(\mathrm{P})$ if and only if there exists a "potential" function $p: V \rightarrow R$ such that

(i) for each $a \in A$,

$$
\begin{aligned}
& \gamma_{p}(a)>0 \quad \Longrightarrow \quad \varphi(a)=\underline{c}(a), \\
& \gamma_{p}(a)<0 \quad \Longrightarrow \quad \varphi(a)=\bar{c}(a),
\end{aligned}
$$

(ii) $\partial \varphi$ maximizes $\omega[p]$, that is, $\omega[p](\partial \varphi) \geq \omega[p](x)$ for any $x \in \mathbf{Z}^{V}$.

(2) Let $p$ be a potential that satisfies (i)-(ii) above for some (optimal) flow $\varphi$. A feasible flow $\varphi^{\prime}$ is optimal if and only if it satisfies (i)-(ii) (with $\varphi$ replaced by $\left.\varphi^{\prime}\right)$.

(Proof) See Section 5.1.

To describe the second criterion we need to introduce an auxiliary network $\mathcal{N}_{\varphi}=\left(G_{\varphi}=\left(V, A_{\varphi}\right), \gamma_{\varphi}\right)$ associated with a feasible flow $\varphi$, which is a slight modification of the standard tool ${ }^{4}$ for the submodular flow problem. The underlying graph $G_{\varphi}$ has the vertex set $V$ and the arc set $A_{\varphi}$ consisting of three disjoint parts: $A_{\varphi}=A_{\varphi}^{*} \cup B_{\varphi}^{*} \cup C_{\varphi}$, where

$$
\begin{aligned}
& A_{\varphi}^{*}=\{a \mid a \in A, \varphi(a)<\bar{c}(a)\} \\
& B_{\varphi}^{*}=\{\bar{a} \mid a \in A, \underline{c}(a)<\varphi(a)\} \quad(\bar{a}: \text { reorientation of } a), \\
& C_{\varphi}=\left\{(u, v) \mid u, v \in V, u \neq v, \partial \varphi-\chi_{u}+\chi_{v} \in B\right\} .
\end{aligned}
$$

The length function $\gamma_{\varphi}: A_{\varphi} \rightarrow R$ is defined by

$$
\gamma_{\varphi}(a)= \begin{cases}\gamma(a) & \left(a \in A_{\varphi}^{*}\right) \\ -\gamma(\bar{a}) & \left(a=(u, v) \in B_{\varphi}^{*}, \bar{a}=(v, u) \in A\right) \\ -\omega(\partial \varphi, u, v) & \left(a=(u, v) \in C_{\varphi}\right)\end{cases}
$$

where $\omega(\partial \varphi, u, v)=\omega\left(\partial \varphi-\chi_{u}+\chi_{v}\right)-\omega(\partial \varphi)$ as in (2.4). We call a directed cycle of negative length a negative cycle.

\footnotetext{
${ }^{4}$ We follow the definition as well as the notation in Fujishige [17], except that the capacity function is not needed here.
} 
Theorem 3.2 A feasible flow $\varphi: A \rightarrow \mathbf{Z}$ is optimal for $(\mathrm{P})$ if and only if there exists no negative cycle in the auxiliary network $\mathcal{N}_{\varphi}=\left(G_{\varphi}, \gamma_{\varphi}\right)$.

(Proof) See Section 5.1.

Remark 3.1 The negative cycle criterion in Theorem 3.2 immediately suggests a primal-type cycle-canceling algorithm for finding an optimal flow, which terminates in a finite number of steps, provided an optimal flow exists.

Remark 3.2 The exchangeability graph $G(x, y)$ with $x=\partial \varphi$ (and any $y$ ), as introduced in Section 2.2, is a subgraph of $G_{\varphi}$. Note, however, that the arc weight is the negative of the arc length.

A sufficient condition for the boundedness of the objective function value $\Gamma(\varphi)$ can be given using a subgraph of $G_{\varphi}$ as follows. Let

$$
\begin{aligned}
& V_{-\infty}=V-\{v \in V \mid \exists K \in R, \forall x \in B: x(v)>K\} \\
& V_{+\infty}=V-\{v \in V \mid \exists K \in R, \forall x \in B: x(v)<K\}
\end{aligned}
$$

and define $\widehat{G}=(V, \widehat{A})$ to be the subgraph of $G_{\varphi}$ with $\widehat{A}=A^{*} \cup B^{*} \cup C^{*}$, where

$$
\begin{aligned}
& A^{*}=\{a \mid a \in A, \bar{c}(a)=+\infty\} \\
& B^{*}=\{\bar{a} \mid a \in A, \underline{c}(a)=-\infty\} \quad(\bar{a}: \text { reorientation of } a), \\
& C^{*}=\left\{(u, v) \in C_{\varphi} \mid u \in V_{-\infty}, v \in V_{+\infty}, u \neq v\right\} .
\end{aligned}
$$

Furthermore let $\widehat{\gamma}_{\varphi}: \widehat{A} \rightarrow R$ be the restriction of the arc length $\gamma_{\varphi}$ of (3.5) to $\widehat{A}$.

Theorem 3.3 If there exists a feasible flow $\varphi$ for $(\mathrm{P})$ such that there is no negative cycle in the network $\left(\widehat{G}, \widehat{\gamma}_{\varphi}\right)$, then there exists $K \in R$ such that $\Gamma\left(\varphi^{\prime}\right) \geq K$ for all feasible flow $\varphi^{\prime}$.

(Proof) See Section 5.2.

As a corollary we obtain a necessary and sufficient condition for the existence of an optimal flow in the case of $R=\mathbf{Z}$.

Theorem 3.4 Suppose $R=\mathbf{Z}$. There exists an optimal flow for $(\mathrm{P})$ if and only if there exists a feasible flow $\varphi$ such that there is no negative cycle in the network $\left(\widehat{G}, \widehat{\gamma}_{\varphi}\right)$.

(Proof) See Section 5.2. 
Remark 3.3 Similar statements in the case of linear cost function can be found in Cunningham-Frank [2, Theorem 8] and Fujishige [17, Theorem 5.5]. It is remarked, however, that the graph $\widehat{G}$ is not identical with the graph, say $\widehat{G}^{\prime}$, employed there. The arc set $C^{*}$ here is replaced in $\widehat{G}^{\prime}$ by

$$
C=\left\{(u, v) \mid u, v \in V, u \neq v, \partial \varphi-\alpha\left(\chi_{u}-\chi_{v}\right) \in B \text { for any } \alpha>0, \alpha \in \mathbf{Z}\right\},
$$

and the arc length $\widehat{\gamma}_{\varphi}$ here depends on $\varphi$ in order to represent the nonlinearity of $\omega$ while it is constant there. In case $\omega$ is trivial $(\omega=0)$ or affine (cf. Example 2.1), the dependence of $\widehat{\gamma}_{\varphi}$ on $\varphi$ disappears, but Theorem 3.4 above does not coincide with the above-mentioned result, which is stronger in two ways; (i) it guarantees the existence of the optimal solution for a general $R$, and (ii) it uses the more natural arc set $C$, which is a subset of $C^{*}$. Note also that $C$ does not depend on $\varphi$ in spite of the apparent dependence in the definition.

\section{Discrete Separation Theorem}

From the optimality criterion (Theorem 3.1) we can derive a discrete separation theorem for a pair of M-concave/convex functions, as follows. Let $\omega: \mathbf{Z}^{V} \rightarrow$ $R \cup\{-\infty\}$ and $\zeta: \mathbf{Z}^{V} \rightarrow R \cup\{+\infty\}$ be such that $\omega$ and $-\zeta$ are M-concave. A discrete separation theorem for such a pair $(\omega, \zeta)$ is given in Theorem 4.2 below. First we note the following corollary to Theorem 3.1.

Theorem 4.1 Assume that $\omega_{1}, \omega_{2}: \mathbf{Z}^{V} \rightarrow R \cup\{-\infty\}$ are $M$-concave functions and let $x^{*} \in \mathbf{Z}^{V}$ be such that $\omega_{i}\left(x^{*}\right) \neq-\infty$ for $i=1,2$. Then $\omega_{1}\left(x^{*}\right)+\omega_{2}\left(x^{*}\right) \geq$ $\omega_{1}(x)+\omega_{2}(x)\left(x \in \mathbf{Z}^{V}\right)$ if and only if there exists $p: V \rightarrow R$ such that $\omega_{1}[-p]\left(x^{*}\right) \geq$ $\omega_{1}[-p](x), \omega_{2}[p]\left(x^{*}\right) \geq \omega_{2}[p](x)\left(x \in \mathbf{Z}^{V}\right)$.

Theorem 4.2 Assume that $\omega$ and $-\zeta$ are $M$-concave functions such that $\omega(x) \neq$ $-\infty$ and $\zeta(x) \neq+\infty$ for some $x \in \mathbf{Z}^{V}$. If $\omega(x) \leq \zeta(x)$ for all $x \in \mathbf{Z}^{V}$, then there exists $p: V \rightarrow R$ and $\alpha \in R$ such that

$$
\omega(x) \leq \alpha+\langle p, x\rangle \leq \zeta(x) \quad\left(x \in \mathbf{Z}^{V}\right) .
$$

(Proof) Take $\omega_{1}=\omega$ and $\omega_{2}=-\zeta$ in Theorem 4.1. Since $\omega_{1}(x)+\omega_{2}(x) \leq 0$ for all $x \in \mathbf{Z}^{V}$, there exists $x=x^{*} \in \mathbf{Z}^{V}$ that maximizes $\omega_{1}(x)+\omega_{2}(x)$. Hence there exists $p: V \rightarrow R$ such that

$$
\begin{aligned}
& \omega(x)-\langle p, x\rangle \leq \omega\left(x^{*}\right)-\left\langle p, x^{*}\right\rangle \quad\left(x \in \mathbf{Z}^{V}\right), \\
& \zeta(x)-\langle p, x\rangle \geq \zeta\left(x^{*}\right)-\left\langle p, x^{*}\right\rangle \quad\left(x \in \mathbf{Z}^{V}\right) .
\end{aligned}
$$

Since $\omega\left(x^{*}\right) \leq \zeta\left(x^{*}\right)$ by the assumption, there exists $\alpha \in R$ with $\omega\left(x^{*}\right)-\left\langle p, x^{*}\right\rangle \leq$ $\alpha \leq \zeta\left(x^{*}\right)-\left\langle p, x^{*}\right\rangle$. This establishes (4.1). 
Remark 4.1 The discrete separation theorem above (Theorem 4.2) should be distinguished from Frank's discrete separation theorem [12] for sub/supermodular functions. A thorough discussion is made in [30], [31] about the relationship to Frank's discrete separation theorem as well as other implications of the present result in the context of "discrete convex analysis." It is also mentioned that Theorem 4.2 above is a straightforward extension of Theorem 3.7 of [27], which gives a similar statement for a pair of matroid valuation and co-valuation based on the optimality criterion [25, Theorems 4.1,4.2] for the valuated matroid intersection problem.

\section{Proofs}

\subsection{Proof of Theorems 3.1 and 3.2}

We are to prove the equivalence of the following three conditions for a feasible flow $\varphi$ :

$(\mathrm{OPT}) \varphi$ is optimal.

(NNC) There is no negative cycle in $\left(G_{\varphi}, \gamma_{\varphi}\right)$.

(POT) There exists a potential $p$ with (i)-(ii) in Theorem 3.1.

We prove $(\mathrm{OPT}) \Rightarrow(\mathrm{NNC}) \Rightarrow(\mathrm{POT}) \Rightarrow(\mathrm{OPT})$ and finally the second part of Theorem 3.1. The proof is an adaptation of the one for the valuated matroid intersection problem in Murota [25].

$(\mathrm{OPT}) \Rightarrow(\mathrm{NNC})$ : Suppose $\left(G_{\varphi}, \gamma_{\varphi}\right)$ has a negative cycle. Let $Q\left(\subseteq A_{\varphi}\right)$ be the arc set of a negative cycle having the smallest number of arcs, and let $\bar{\varphi}: A \rightarrow \mathbf{Z}$ be defined by

$$
\bar{\varphi}(a)= \begin{cases}\varphi(a)+1 & \left(a \in Q \cap A_{\varphi}^{*}\right) \\ \varphi(\bar{a})-1 & \left(a=(u, v) \in Q \cap B_{\varphi}^{*}, \bar{a}=(v, u) \in A\right) \\ \varphi(a) & \text { (otherwise) }\end{cases}
$$

Note that $|\partial \varphi(v)-\partial \bar{\varphi}(v)| \leq 1(v \in V)$.

Lemma $5.1(\partial \varphi, \partial \bar{\varphi})$ satisfies the unique-max condition.

(Proof) We make use of an extension of Fujishige's proof technique [15], [17, Lemma 5.4]. Consider the bipartite graph $G(\partial \varphi, \partial \bar{\varphi})=\left(V^{+}, V^{-} ; \widehat{A}\right)$ with $V^{+}=$ $\operatorname{supp}^{+}(\partial \varphi-\partial \bar{\varphi}), V^{-}=\operatorname{supp}^{-}(\partial \varphi-\partial \bar{\varphi})$ and

$$
\widehat{A}=\left\{(u, v) \mid u \in V^{+}, v \in V^{-}, x-\chi_{u}+\chi_{v} \in B\right\},
$$


and take a maximum-weight perfect matching $M=\left\{\left(u_{i}, v_{i}\right) \mid i=1, \cdots, m\right\}$ (where $m=\|\partial \varphi-\partial \bar{\varphi}\| / 2)$ in $G(\partial \varphi, \partial \bar{\varphi})$ with respect to the arc weight $\omega(\partial \varphi, u, v)$ as well as the potential function $\widehat{p}$ as in Lemma 2.6(1). Then $M$ is a subset of

$$
C_{\varphi}^{*}=\left\{(u, v) \mid u \in V^{+}, v \in V^{-}, \omega(\partial \varphi, u, v)-\widehat{p}(u)+\widehat{p}(v)=0\right\} .
$$

Put $Q^{\prime}=\left(Q-C_{\varphi}\right) \cup M$, where $M$ is now regarded as a subset of $C_{\varphi}$ as in Remark 3.2. $Q^{\prime}$ is a disjoint union of cycles in $G_{\varphi}$ with its length

$$
\gamma_{\varphi}\left(Q^{\prime}\right)=\gamma_{\varphi}(Q)+\left[\gamma_{\varphi}(M)-\gamma_{\varphi}\left(Q \cap C_{\varphi}\right)\right]
$$

being negative, since $\gamma_{\varphi}(Q)<0$ and $\left[\gamma_{\varphi}(M)-\gamma_{\varphi}\left(Q \cap C_{\varphi}\right)\right] \leq 0$, the latter of which follows from the observation that $-\gamma_{\varphi}(M)$ is equal to the maximum weight of a perfect matching in $G(\partial \varphi, \partial \bar{\varphi})$ and $Q \cap C_{\varphi}$ can be identified with a perfect matching in $G(\partial \varphi, \partial \bar{\varphi})$. The minimality of $Q$ (with respect to the number of arcs) implies that $Q^{\prime}$ itself is a negative cycle having the smallest number of arcs.

Suppose, to the contrary, that $(\partial \varphi, \partial \bar{\varphi})$ does not satisfy the unique-max condition. Since $\left(u_{i}, v_{i}\right) \in C_{\varphi}^{*}$ for $i=1, \cdots, m$, it follows from Lemma 2.6(2) that there are distinct indices $i_{k}(k=1, \cdots, q ; q \geq 2)$ such that $\left(u_{i_{k}}, v_{i_{k+1}}\right) \in C_{\varphi}^{*}$ for $k=1, \cdots, q$, where $i_{q+1}=i_{1}$. That is,

$$
\omega\left(\partial \varphi, u_{i_{k}}, v_{i_{k+1}}\right)=\widehat{p}\left(u_{i_{k}}\right)-\widehat{p}\left(v_{i_{k+1}}\right) \quad(k=1, \cdots, q) .
$$

On the other hand we have

$$
\omega\left(\partial \varphi, u_{i_{k}}, v_{i_{k}}\right)=\widehat{p}\left(u_{i_{k}}\right)-\widehat{p}\left(v_{i_{k}}\right) \quad(k=1, \cdots, q) .
$$

It then follows that

$$
\sum_{k=1}^{q} \omega\left(\partial \varphi, u_{i_{k}}, v_{i_{k+1}}\right)=\sum_{k=1}^{q} \omega\left(\partial \varphi, u_{i_{k}}, v_{i_{k}}\right) \quad\left(=\sum_{k=1}^{q}\left[\widehat{p}\left(u_{i_{k}}\right)-\widehat{p}\left(v_{i_{k}}\right)\right]\right)
$$

i.e.,

$$
\sum_{k=1}^{q} \gamma_{\varphi}\left(u_{i_{k}}, v_{i_{k+1}}\right)=\sum_{k=1}^{q} \gamma_{\varphi}\left(u_{i_{k}}, v_{i_{k}}\right)
$$

For $k=1, \cdots, q$, let $P^{\prime}\left(v_{i_{k+1}}, u_{i_{k}}\right)$ denote the path on $Q^{\prime}$ from $v_{i_{k+1}}$ to $u_{i_{k}}$, and let $Q_{k}^{\prime}$ be the directed cycle formed by arc $\left(u_{i_{k}}, v_{i_{k+1}}\right)$ and path $P^{\prime}\left(v_{i_{k+1}}, u_{i_{k}}\right)$. Obviously,

$$
\gamma_{\varphi}\left(Q_{k}^{\prime}\right)=\gamma_{\varphi}\left(u_{i_{k}}, v_{i_{k+1}}\right)+\gamma_{\varphi}\left(P^{\prime}\left(v_{i_{k+1}}, u_{i_{k}}\right)\right) \quad(k=1, \cdots, q) .
$$

A simple but crucial observation here is that

$$
\left(\bigcup_{k=1}^{q} P^{\prime}\left(v_{i_{k+1}}, u_{i_{k}}\right)\right) \cup\left\{\left(u_{i_{k}}, v_{i_{k}}\right) \mid k=1, \cdots, q\right\}=q^{\prime} \cdot Q^{\prime}
$$


for some $q^{\prime}$ with $1 \leq q^{\prime}<q$, where the union denotes the multiset union, and this expression means that each element of $Q^{\prime}$ appears $q^{\prime}$ times on the left hand side. Hence by adding (5.6) over $k=1, \cdots, q$ we obtain

$$
\begin{aligned}
\sum_{k=1}^{q} \gamma_{\varphi}\left(Q_{k}^{\prime}\right) & =\sum_{k=1}^{q} \gamma_{\varphi}\left(u_{i_{k}}, v_{i_{k+1}}\right)+\sum_{k=1}^{q} \gamma_{\varphi}\left(P^{\prime}\left(v_{i_{k+1}}, u_{i_{k}}\right)\right) \\
& =\left[\sum_{k=1}^{q} \gamma_{\varphi}\left(u_{i_{k}}, v_{i_{k+1}}\right)-\sum_{k=1}^{q} \gamma_{\varphi}\left(u_{i_{k}}, v_{i_{k}}\right)\right]+q^{\prime} \cdot \gamma_{\varphi}\left(Q^{\prime}\right) \\
& =q^{\prime} \cdot \gamma_{\varphi}\left(Q^{\prime}\right)<0,
\end{aligned}
$$

where the last equality is due to (5.5). This implies that $\gamma_{\varphi}\left(Q_{k}^{\prime}\right)<0$ for some $k$, while $Q_{k}^{\prime}$ has a smaller number of arcs than $Q^{\prime}$. This contradicts the minimality of $Q^{\prime}$. Therefore $(\partial \varphi, \partial \bar{\varphi})$ satisfies the unique-max condition.

Lemma 5.2 For a negative cycle $Q$ in $G_{\varphi}$ having the smallest number of arcs, $\bar{\varphi}$ defined by (5.1) is a feasible flow with $\Gamma(\bar{\varphi}) \leq \Gamma(\varphi)+\gamma_{\varphi}(Q)(<\Gamma(\varphi))$.

(Proof) By Lemma 5.1 and Lemma 2.5 ("unique-max lemma") we have

$$
\omega(\partial \bar{\varphi})=\omega(\partial \varphi)+\widehat{\omega}(\partial \varphi, \partial \bar{\varphi}) \geq \omega(\partial \varphi)-\gamma_{\varphi}\left(Q \cap C_{\varphi}\right)
$$

Also we have

$$
\langle\gamma, \bar{\varphi}\rangle=\langle\gamma, \varphi\rangle+\gamma_{\varphi}\left(Q \cap\left(A_{\varphi}^{*} \cup B_{\varphi}^{*}\right)\right) .
$$

These inequalities, when subtracted, yield $\Gamma(\bar{\varphi}) \leq \Gamma(\varphi)+\gamma_{\varphi}(Q)$.

The above lemma shows "(OPT) $\Rightarrow(\mathrm{NNC})$ ".

$(\mathrm{NNC}) \Rightarrow(\mathrm{POT})$ : By the well-known fact in graph theory, (NNC) implies the existence of a function $p: V \rightarrow R$ such that $\gamma_{\varphi}(a)+p\left(\partial^{+} a\right)-p\left(\partial^{-} a\right) \geq 0\left(a \in A_{\varphi}\right)$. This condition for $a \in A_{\varphi}^{*} \cup B_{\varphi}^{*}$ is equivalent to the condition (i) in Theorem 3.1. For $a=(u, v) \in C_{\varphi}$, on the other hand, it means

$$
\omega[p](\partial \varphi, u, v)=\omega[p]\left(\partial \varphi-\chi_{u}+\chi_{v}\right)-\omega[p](\partial \varphi)=\omega(\partial \varphi, u, v)-p(u)+p(v) \leq 0
$$

This implies the condition (ii) by Theorem 2.3. Thus "(NNC) $\Rightarrow(\mathrm{POT})$ " has been shown.

$(\mathrm{POT}) \Rightarrow(\mathrm{OPT}):$ For any $\varphi: A \rightarrow \mathbf{Z}$ and $p: V \rightarrow R$ we have

$$
\begin{aligned}
\Gamma(\varphi) & =\langle\gamma, \varphi\rangle-\omega(\partial \varphi) \\
& =\left\langle\gamma_{p}, \varphi\right\rangle-(\omega(\partial \varphi)+\langle p, \partial \varphi\rangle) \\
& =\left\langle\gamma_{p}, \varphi\right\rangle-\omega[p](\partial \varphi) .
\end{aligned}
$$


Suppose $\varphi$ and $p$ satisfy (i)-(ii) of Theorem 3.1, and take an arbitrary feasible flow $\varphi^{\prime}$. Since

$$
\begin{aligned}
& \left\langle\gamma_{p}, \varphi^{\prime}-\varphi\right\rangle \\
& =\sum_{a: \gamma_{p}(a)>0} \gamma_{p}(a)\left(\varphi^{\prime}(a)-\underline{c}(a)\right)+\sum_{a: \gamma_{p}(a)<0} \gamma_{p}(a)\left(\varphi^{\prime}(a)-\bar{c}(a)\right) \geq 0,
\end{aligned}
$$

we have

$$
\Gamma\left(\varphi^{\prime}\right)=\left\langle\gamma_{p}, \varphi^{\prime}\right\rangle-\omega[p]\left(\partial \varphi^{\prime}\right) \geq\left\langle\gamma_{p}, \varphi\right\rangle-\omega[p](\partial \varphi)=\Gamma(\varphi)
$$

This shows that $\varphi$ is optimal, establishing "(POT) $\Rightarrow(\mathrm{OPT})$ ".

Finally for the second half of Theorem 3.1 we note in the above inequality that $\Gamma\left(\varphi^{\prime}\right)=\Gamma(\varphi)$ if and only if $\left\langle\gamma_{p}, \varphi^{\prime}\right\rangle=\left\langle\gamma_{p}, \varphi\right\rangle$ and $\omega[p]\left(\partial \varphi^{\prime}\right)=\omega[p](\partial \varphi)$. From (5.7) we note further that $\left\langle\gamma_{p}, \varphi^{\prime}\right\rangle=\left\langle\gamma_{p}, \varphi\right\rangle$ is equivalent to the condition (i) for $\varphi^{\prime}$. We have completed the proofs of Theorem 3.1 and Theorem 3.2.

\subsection{Proof of Theorems 3.3 and 3.4}

Take a feasible flow $\varphi$ such that there is no negative cycle in $\left(\widehat{G}, \widehat{\gamma}_{\varphi}\right)$. Then there exists $p: V \rightarrow R$, depending on $\varphi$, such that $\widehat{\gamma}_{\varphi}(a)+p\left(\partial^{+} a\right)-p\left(\partial^{-} a\right) \geq 0(a \in \widehat{A})$. This is equivalent to the following set of conditions:

$$
\begin{aligned}
\underline{c}(a)=-\infty & \Longrightarrow \gamma_{p}(a) \leq 0, \\
\bar{c}(a)=+\infty & \Longrightarrow \gamma_{p}(a) \geq 0, \\
u \in V_{-\infty}, v \in V_{+\infty}, \partial \varphi-\chi_{u}+\chi_{v} \in B & \Longrightarrow \omega[p](\partial \varphi, u, v) \leq 0 .
\end{aligned}
$$

The first two conditions imply that there exists $K_{1} \in R$ (depending on $\varphi$ ) such that

$$
\left\langle\gamma_{p}, \varphi^{\prime}\right\rangle \geq K_{1} \quad\left(\forall \varphi^{\prime}: \text { feasible flow }\right)
$$

The last condition shows, on the other hand, that there exists $K_{2} \in R$ (depending on $\varphi$ ) such that (cf. (2.7))

$$
\widehat{\omega[p]}\left(\partial \varphi, \partial \varphi^{\prime}\right) \leq K_{2} \quad\left(\forall \varphi^{\prime}: \text { feasible flow }\right)
$$

which in turn implies, by Lemma 2.4 ("upper-bound lemma"), that

$$
\omega[p]\left(\partial \varphi^{\prime}\right) \leq \omega[p](\partial \varphi)+K_{2} \quad\left(\forall \varphi^{\prime}: \text { feasible flow }\right)
$$

Therefore we obtain

$$
\Gamma\left(\varphi^{\prime}\right)=\left\langle\gamma_{p}, \varphi^{\prime}\right\rangle-\omega[p]\left(\partial \varphi^{\prime}\right) \geq K_{1}-K_{2}-\omega[p](\partial \varphi) \quad\left(\forall \varphi^{\prime}: \text { feasible flow }\right) .
$$

This establishes Theorem 3.3. 
The sufficiency in Theorem 3.4 follows from Theorem 3.3 and the observation that in the case of $R=\mathbf{Z}$ the boundedness (from below) of $\Gamma(\varphi)$ is equivalent to the existence of an optimal flow. The necessity follows from Theorem 3.2 since for optimal $\varphi$ there is no negative cycle in $\left(G_{\varphi}, \gamma_{\varphi}\right)$, a fortiori nor in $\left(\widehat{G}, \widehat{\gamma}_{\varphi}\right)$. The proof of Theorem 3.4 is completed.

\section{Acknowledgements}

The author thanks Satoru Fujishige and Satoru Iwata for valuable comments on the manuscript. He is also indebted to Bill Cunningham and Satoru Fujishige for the information about the exchange property of base polyhedra.

\section{A Appendix}

\section{A.1 Reduction of arc cost to boundary cost}

We transform the problem:

Minimize $\quad \Gamma_{2}(\varphi)=-\omega_{A}(\varphi)-\omega(\partial \varphi)$

subject to

$$
\begin{aligned}
& \underline{c}(a) \leq \varphi(a) \leq \bar{c}(a) \quad(a \in A), \\
& \partial \varphi \in B
\end{aligned}
$$

defined for $G=(V, A)$ into a problem of the form (P) for an enlarged graph $G^{\prime}=\left(V^{\prime}, A^{\prime}\right)$. The idea is to split each arc $a=(u, w) \in A$ into two copies $a^{+}=\left(u, v_{a}^{-}\right) \in A^{\prime}, a^{-}=\left(v_{a}^{+}, w\right) \in A^{\prime}$ by introducing new vertices $v_{a}^{-}, v_{a}^{+} \in V^{\prime}$, and to convert the flow in $a \in A$ into the boundary at $v_{a}^{+} \in V^{\prime}$. Putting

$$
\begin{array}{ll}
V^{+}=\left\{v_{a}^{+} \mid a \in A\right\}, & V^{-}=\left\{v_{a}^{-} \mid a \in A\right\}, \\
A^{+}=\left\{a^{+} \mid a \in A\right\}, & A^{-}=\left\{a^{-} \mid a \in A\right\},
\end{array}
$$

we define

$$
\begin{aligned}
& V^{\prime}=V \cup V^{+} \cup V^{-}, \quad A^{\prime}=A^{+} \cup A^{-}, \\
& \bar{c}^{\prime}\left(a^{+}\right)=\bar{c}^{\prime}\left(a^{-}\right)=\bar{c}(a), \quad \underline{c}^{\prime}\left(a^{+}\right)=\underline{c}^{\prime}\left(a^{-}\right)=\underline{c}(a) \quad(a \in A), \\
& \gamma^{\prime}\left(a^{+}\right)=\gamma^{\prime}\left(a^{-}\right)=0 \quad(a \in A), \\
& B^{\prime}=\left\{\left(x, y^{+}, y^{-}\right) \mid x \in B, y^{+}+y^{-}=0\left(x \in \mathbf{Z}^{V}, y^{+} \in \mathbf{Z}^{V^{+}}, y^{-} \in \mathbf{Z}^{V^{-}}\right)\right\} \\
& \omega^{\prime}\left(x, y^{+}, y^{-}\right)=\omega(x)+\omega_{A}\left(y^{+}\right) \quad\left(x \in \mathbf{Z}^{V}, y^{+} \in \mathbf{Z}^{V^{+}}, y^{-} \in \mathbf{Z}^{V^{-}}\right) .
\end{aligned}
$$


It can be shown as in Example 2.2 that if $\omega$ is M-concave and $\omega_{A}$ is separable concave, i.e., $\omega_{A}(\varphi)=\sum_{a \in A} g_{a}(\varphi(a))$ with $g_{a}$ being concave for $a \in A$, then $\omega^{\prime}$ is also M-concave, satisfying (EXC). Then the problem (P) defined by the primed data is equivalent to the given problem.

\section{A.2 Proof of Theorem 2.2}

We prove $\left(\mathrm{EXC}_{\mathrm{w}}\right) \Longrightarrow(\mathrm{EXC})$. For $p: V \rightarrow R$ we abbreviate $\omega[p]$ of (3.2) to $\omega_{p}$. As in (2.4) we put $\omega_{p}(x, u, v)=\omega_{p}(x-u+v)-\omega_{p}(x)$. Define $B=\left\{x \in \mathbf{Z}^{V}\right.$ $\omega(x) \neq-\infty\}$. If $x, y \in B$, we have

$$
\omega(x, u, v)+\omega(y, v, u)=\omega_{p}(x, u, v)+\omega_{p}(y, v, u) .
$$

Lemma A.1 Let $x \in B, y=x-\chi_{u_{0}}-\chi_{u_{1}}+\chi_{v_{0}}+\chi_{v_{1}}$ with $u_{0}, u_{1}, v_{0}, v_{1} \in V$ and $\left\{u_{0}, u_{1}\right\} \cap\left\{v_{0}, v_{1}\right\}=\emptyset$, and let $p: V \rightarrow R$. If $\left(\mathrm{EXC}_{\mathrm{w}}\right)$ is satisfied, then

$$
\omega_{p}(y)-\omega_{p}(x) \leq \max \left(\pi_{00}+\pi_{11}, \pi_{01}+\pi_{10}\right),
$$

where $\pi_{i j}=\omega_{p}\left(x, u_{i}, v_{j}\right)$ for $i, j=0,1$.

(Proof) By $\left(\mathrm{EXC}_{\mathrm{w}}\right)$ we have

$$
\omega(y)-\omega(x) \leq \max \left(\omega\left(x, u_{0}, v_{0}\right)+\omega\left(x, u_{1}, v_{1}\right), \omega\left(x, u_{0}, v_{1}\right)+\omega\left(x, u_{1}, v_{0}\right)\right) .
$$

The desired inequality follows from this.

Define

$$
\begin{gathered}
\mathcal{D}=\left\{(x, y) \mid x, y \in B, \exists u_{*} \in \operatorname{supp}^{+}(x-y), \forall v \in \operatorname{supp}^{-}(x-y):\right. \\
\left.\omega\left(x, u_{*}, v\right)+\omega\left(y, v, u_{*}\right)<0\right\},
\end{gathered}
$$

which denotes the set of pairs $(x, y)$ for which the exchangeability in (EXC) fails. We are to show $\mathcal{D}=\emptyset$.

Suppose to the contrary that $\mathcal{D} \neq \emptyset$, and take $(x, y) \in \mathcal{D}$ such that $\|x-y\|$ is minimum and let $u_{*} \in \operatorname{supp}^{+}(x-y)$ be as in the definition of $\mathcal{D}$. Define $p: V \rightarrow R$ by

$p(v)= \begin{cases}-\omega\left(x, u_{*}, v\right) & \left(v \in \operatorname{supp}^{-}(x-y), x-\chi_{u_{*}}+\chi_{v} \in B\right) \\ \omega\left(y, v, u_{*}\right)+\varepsilon & \left(v \in \operatorname{supp}^{-}(x-y), x-\chi_{u_{*}}+\chi_{v} \notin B, y+\chi_{u_{*}}-\chi_{v} \in B\right) \\ 0 & \text { (otherwise) }\end{cases}$

with some $\varepsilon>0$ and consider $\omega_{p}$.

\section{Claim 1:}

$$
\begin{array}{ll}
\omega_{p}\left(x, u_{*}, v\right)=0 & \text { if } v \in \operatorname{supp}^{-}(x-y), x-\chi_{u_{*}}+\chi_{v} \in B \\
\omega_{p}\left(y, v, u_{*}\right)<0 & \text { for } v \in \operatorname{supp}^{-}(x-y) .
\end{array}
$$


The equality (A.2) is immediate from the definitions. The inequality (A.3) can be shown as follows. If $x-\chi_{u_{*}}+\chi_{v} \in B$, we have $\omega_{p}\left(x, u_{*}, v\right)=0$ by (A.2) and

$$
\omega_{p}\left(x, u_{*}, v\right)+\omega_{p}\left(y, v, u_{*}\right)=\omega\left(x, u_{*}, v\right)+\omega\left(y, v, u_{*}\right)<0
$$

by (A.1) and the definition of $u_{*}$. Otherwise we have $\omega_{p}\left(y, v, u_{*}\right)=-\varepsilon$ or $-\infty$ according to whether $y+\chi_{u_{*}}-\chi_{v} \in B$ or not.

Claim 2: There exist $u_{0} \in \operatorname{supp}^{+}(x-y)$ and $v_{0} \in \operatorname{supp}^{-}(x-y)$ such that $u_{0} \neq u_{*}, y+\chi_{u_{0}}-\chi_{v_{0}} \in B$, and

$$
\omega_{p}\left(y, v_{0}, u_{0}\right) \geq \omega_{p}\left(y, v, u_{0}\right) \quad\left(v \in \operatorname{supp}^{-}(x-y)\right) .
$$

In fact, $\left(\mathrm{EXC}_{\mathrm{w}}\right)$ implies that $\exists u_{0} \in \operatorname{supp}^{+}(x-y), \exists v_{0} \in \operatorname{supp}^{-}(x-y)$ with

$$
-\infty \neq \omega(x)+\omega(y) \leq \omega\left(x-\chi_{u_{0}}+\chi_{v_{0}}\right)+\omega\left(y+\chi_{u_{0}}-\chi_{v_{0}}\right) .
$$

We have $u_{0} \neq u_{*}$ by the definition of $u_{*}$, and $y+\chi_{u_{0}}-\chi_{v_{0}} \in B$ by $\omega\left(y+\chi_{u_{0}}-\chi_{v_{0}}\right) \neq$ $-\infty$. Fixing such $u_{0}$ and choosing $v_{0}$ appropriately, we can further assume (A.4).

Put $y^{\prime}=y+\chi_{u_{0}}-\chi_{v_{0}}$.

Claim 3: $\left(x, y^{\prime}\right) \in \mathcal{D}$.

To prove this it suffices to show

$$
\omega_{p}\left(x, u_{*}, v\right)+\omega_{p}\left(y^{\prime}, v, u_{*}\right)<0 \quad\left(v \in \operatorname{supp}^{-}\left(x-y^{\prime}\right)\right) .
$$

We may restrict ourselves to $v$ with $x-\chi_{u_{*}}+\chi_{v} \in B$, since otherwise the first term $\omega_{p}\left(x, u_{*}, v\right)$ is equal to $-\infty$. For such $v$ the first term is equal to zero by (A.2). For the second term it follows from Lemma A.1, (A.3) and (A.4) that

$$
\begin{aligned}
\omega_{p}\left(y^{\prime}, v, u_{*}\right) & =\omega_{p}\left(y+\chi_{u_{0}}+\chi_{u_{*}}-\chi_{v_{0}}-\chi_{v}\right)-\omega_{p}\left(y+\chi_{u_{0}}-\chi_{v_{0}}\right) \\
\leq & \max \left[\omega_{p}\left(y, v_{0}, u_{0}\right)+\omega_{p}\left(y, v, u_{*}\right), \omega_{p}\left(y, v, u_{0}\right)+\omega_{p}\left(y, v_{0}, u_{*}\right)\right] \\
& \quad-\omega_{p}\left(y, v_{0}, u_{0}\right) \\
< & \max \left[\omega_{p}\left(y, v_{0}, u_{0}\right), \omega_{p}\left(y, v, u_{0}\right)\right]-\omega_{p}\left(y, v_{0}, u_{0}\right) \\
& =0 .
\end{aligned}
$$

Since $\left\|x-y^{\prime}\right\|=\|x-y\|-2$, Claim 3 contradicts our choice of $(x, y) \in \mathcal{D}$. Therefore we conclude $\mathcal{D}=\emptyset$, completing the proof of Theorem 2.2.

\section{A.3 Proof of $(\mathrm{B} 2) \Rightarrow(\mathrm{B} 3)$ in Lemma 2.1}

We first note the following easy lemma.

Lemma A.2 Suppose $B \subseteq \mathbf{Z}^{V}$ satisfies (B2). Let $x \in B$, and $y=x-\chi_{u_{0}}-\chi_{u_{1}}+$ $\chi_{v_{0}}+\chi_{v_{1}} \in B$ with $u_{0}, u_{1}, v_{0}, v_{1} \in V$. Then we have either $\left[x-\chi_{u_{0}}+\chi_{v_{0}} \in B\right.$ and $\left.x-\chi_{u_{1}}+\chi_{v_{1}} \in B\right]$ or $\left[x-\chi_{u_{0}}+\chi_{v_{1}} \in B\right.$ and $\left.x-\chi_{u_{1}}+\chi_{v_{0}} \in B\right]$. 
Define

$$
\begin{gathered}
\mathcal{D}=\left\{(x, y) \mid x, y \in B, \exists u_{*} \in \operatorname{supp}^{+}(x-y), \forall v \in \operatorname{supp}^{-}(x-y):\right. \\
\left.x-\chi_{u_{*}}+\chi_{v} \notin B \text { or } y-\chi_{v}+\chi_{u_{*}} \notin B\right\},
\end{gathered}
$$

which denotes the set of pairs $(x, y)$ for which the exchangeability in (B3) fails. We are to show $\mathcal{D}=\emptyset$.

Suppose to the contrary that $\mathcal{D} \neq \emptyset$, and take $(x, y) \in \mathcal{D}$ such that $\|x-y\|$ is minimum and let $u_{*} \in \operatorname{supp}^{+}(x-y)$ be as in the definition of $\mathcal{D}$.

(B2) implies that $\exists v_{0} \in \operatorname{supp}^{-}(x-y), \exists u_{0} \in \operatorname{supp}^{+}(x-y)$ with $x-\chi_{u_{*}}+\chi_{v_{0}} \in B$ and $y+\chi_{u_{0}}-\chi_{v_{0}} \in B$. We have $u_{0} \neq u_{*}$ by the definition of $u_{*}$. Put $y^{\prime}=$ $y+\chi_{u_{0}}-\chi_{v_{0}}$.

Claim: $\left(x, y^{\prime}\right) \in \mathcal{D}$.

To see this, first note that $u_{*} \in \operatorname{supp}^{+}\left(x-y^{\prime}\right)\left(\right.$ since $\left.u_{0} \neq u_{*}\right)$ and consider $v \in$ $\operatorname{supp}^{-}\left(x-y^{\prime}\right)$ with $x-\chi_{u_{*}}+\chi_{v} \in B$. Since $\operatorname{supp}^{-}\left(x-y^{\prime}\right) \subseteq \operatorname{supp}^{-}(x-y)$, we have $y-\chi_{v}+\chi_{u_{*}} \notin B$ as well as $y-\chi_{v_{0}}+\chi_{u_{*}} \notin B$ by the definition of $u_{*}$. Then Lemma A.2 (or its contraposition) implies that $y^{\prime}-\chi_{v}+\chi_{u_{*}}=y-\chi_{v_{0}}-\chi_{v}+\chi_{u_{0}}+\chi_{u_{*}} \notin B$. This establishes the Claim.

However, the Claim contradicts our choice of $(x, y) \in \mathcal{D}$, since $\left\|x-y^{\prime}\right\|=$ $\|x-y\|-2$. Therefore we conclude $\mathcal{D}=\emptyset$, completing the proof of (B2) $\Rightarrow$ (B3). 


\section{References}

[1] R. E. Bixby and W. H. Cunningham: Matroid optimization and algorithms, in: R. L. Graham, M. Grötschel and L. Lovász, eds., Handbook of combinatorics, Vol. I, Elsevier Science B. V., Amsterdam, Chapter 11, pp. 551-609, 1995.

[2] W. H. Cunningham and A. Frank: A primal-dual algorithm for submodular flows, Mathematics of Operations Research 10 (1985), 251-262.

[3] A. W. M. Dress and W. Terhalle: Well-layered maps - A class of greedily optimizable set functions, Applied Mathematics Letters 8 (1995), 77-80.

[4] A. W. M. Dress AND W. Terhalle: Well-layered maps and the maximum-degree $k \times k$-subdeterminant of a matrix of rational functions, $A p$ plied Mathematics Letters 8 (1995), 19-23.

[5] A. W. M. Dress and W. Terhalle: Rewarding maps - On greedy optimization of set functions, Advances in Applied Mathematics, to appear.

[6] A. W. M. Dress and W. Wenzel: Valuated matroid: A new look at the greedy algorithm, Applied Mathematics Letters 3 (1990), 33-35.

[7] A. W. M. Dress And W. Wenzel: Valuated matroids, Advances in Mathematics 93 (1992), 214-250.

[8] J. EDmonds: Submodular functions, matroids and certain polyhedra, in Combinatorial Structures and Their Applications (R. Guy, H. Hanai, N. Sauer and J. Schönsheim, eds.), Gordon and Breach, New York, pp. 69-87, 1970.

[9] J. Edmonds And R. Giles: A min-max relation for submodular functions on graphs, Annals of Discrete Mathematics 1 (1977), 185-204.

[10] U. FAIgle: Matroids in combinatorial optimization, in Combinatorial Geometries (N. White, ed.), Cambridge University Press, London, pp. 161-210, 1987.

[11] A. FRANK: A weighted matroid intersection algorithm, Journal of Algorithms 2 (1981), 328-336.

[12] A. Frank: An algorithm for submodular functions on graphs, Annals of Discrete Mathematics 16 (1982), 97-120. 
[13] A. Frank: Submodular flows, in Progress in Combinatorial Optimization (W. R. Pulleyblank, ed.), Academic Press, New York, pp. 147-165, 1984.

[14] A. Frank And E. TARdos: Generalized polymatroids and submodular flows, Mathematical Programming 42 (1988), 489-563.

[15] S. FuJishige: Algorithms for solving the independent-flow problems, Journal of the Operations Research Society of Japan 21 (1978), 189-204.

[16] S. FuJishige: Structures of polyhedra determined by submodular functions on crossing families, Mathematical Programming 29 (1984), 125-141.

[17] S. Fujishige: Submodular Functions and Optimization, North-Holland, 1991

[18] S. Fujishige, H. Röck And U. Zimmermann: A strongly polynomial algorithm for minimum cost submodular flow problems, Mathematics of $O p$ erations Research 14 (1989), 60-69.

[19] H. N. Gabow: A framework for cost-scaling algorithms for submodular flow problems, in Proc. 34th IEEE Annual Symposium on the Foundations of Computer Science, pp. 449-458, 1993.

[20] R. Hassin: Minimum cost flow with set-constraints, Networks 12 (1982), $1-12$.

[21] E. L. LaWler and C. U. Martel: Computing maximal polymatroidal network flows. Mathematics of Operations Research 7 (1982), 334-347.

[22] C. L. Lucchesi And D. H. Younger: A minimax relation for directed graphs, Journal of the London Mathematical Society 17 (1978), 369-374.

[23] M. Minoux: Solving integer minimum cost flows with separable convex objective polynomially, Mathematical Programming 26 (1986), 237-239.

[24] K. Murota: Finding optimal minors of valuated bimatroids, Applied Mathematics Letters 8 (1995), 37-42.

[25] K. Murota: Valuated matroid intersection, I: optimality criteria, SIAM Journal on Discrete Mathematics 9 (1996), No.3, to appear.

[26] K. Murota: Valuated matroid intersection, II: algorithms, SIAM Journal on Discrete Mathematics 9 (1996), No.3, to appear.

[27] K. Murota: Fenchel-type duality for matroid valuations, Report No. 95839OR, Forschungsinstitut für Diskrete Mathematik, Universität Bonn, 1995. 
[28] K. Murota: On exchange axioms for valuated matroids and valuated deltamatroids, Combinatorica, to appear.

[29] K. Murota: Matroid valuation on independent sets, Report No. 95842-OR, Forschungsinstitut für Diskrete Mathematik, Universität Bonn, 1995.

[30] K. Murota: Convexity and Steinitz's exchange property, Report No. 95848OR, Forschungsinstitut für Diskrete Mathematik, Universität Bonn, 1995. Extended abstract to appear in Proc. Integer Programming and Combinatorial Optimization V, June 1996.

[31] K. Murota: Discrete convex analysis, RIMS preprint, Kyoto University, 1996.

[32] G. L. Nemhauser, A. H. G. Rinnooy Kan and M. J. Todd, Eds.: Optimization, Handbooks in Operations Research and Management Science, Vol. 1, Elsevier Science Publishers, Amsterdam, 1989.

[33] P. Schönsleben: Ganzzahlige Polymatroid - Intersektions - Algorithmen, Dissertation, Eidgenössische Technische Hochschule Zürich, 1980.

[34] A. Schrijver: Total dual integrality from directed graphs, crossing families, and sub- and supermodular functions, in Progress in Combinatorial Optimization (W. R. Pulleyblank, ed.), Academic Press, New York, pp. 315-361, 1984.

[35] N. Tomizawa: Theory of hyperspaces (I) - supermodular functions and generalization of concept of 'bases' (in Japanese). Papers of the Technical Group on Circuit and System Theory, Institute of Electronics and Communication Engineers of Japan, CAS80-72 (1980).

[36] U. Zimmermann: Minimization on submodular flows, Discrete Applied Mathematics 4 (1982), 303-323.

[37] U. Zimmermann: Negative circuits for flows and submodular flows, Discrete Applied Mathematics 36 (1992), 179-189. 